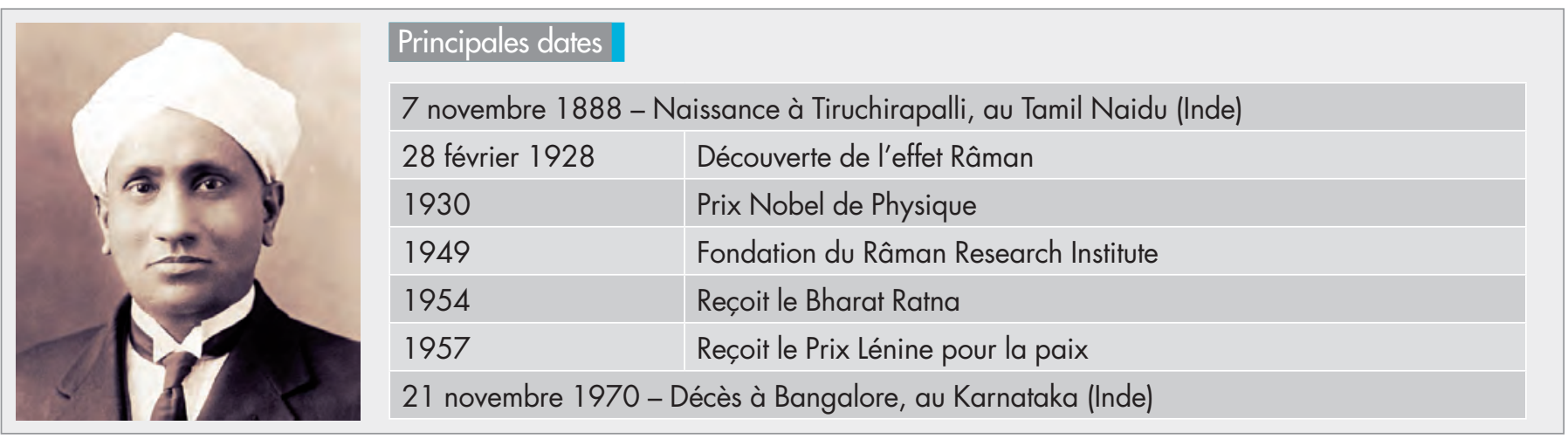

\title{
Chandrashekhara Venkata Râman
}

\section{Riad HAÏDAR}

haidar@onera.fr

\section{Physicien indien, lauréat du prix Nobel de physique en 1930, Sir Chandrashekhara Venkata Râman a découvert et expliqué la diffusion optique inélastique, un effet qui porte désormais son nom.}

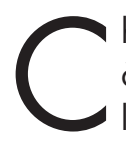
handrasekhara Venkata Râman naît le 7 novembre 1888 à Tiruchirapalli, une grande ville de l'Inde située dans I'État du Tamil Nadu. Il est issu d'une famille Brahmane, la caste traditionnelle des lettrés en Inde. Ses ancêtres étaient des agriculteurs établis dans le district de Thanjavur. Son père, Chandrasekhara lyer, a étudié à Kumbakonam et obtenu son matriculation examination (l'équivalent du baccalauréat français) en 1881 ; il a épousé Parvathi Ammal peu de temps après. Râman est le second de leurs huit enfants. Il est âgé de 4 ans lorsque la famille s'installe à Visakhapatnam où son père, qui a obtenu un Bachelor of Arts en physique en 1891, est nommé au poste de professeur en sciences au College A.V. Narasimha. Plutôt chétif et d'une constitution fragile, Râman possède en revanche des aptitudes intellectuelles peu communes. Admis au College où enseigne son père, il excelle dans les études et décroche tous les prix. II manifeste très tôt un intérêt prononcé pour les sciences physiques, qui se confirme lorsqu'il intègre le prestigieux et très sélectif Presidency College de I'ancienne Madras (actuellement Chennai). Râman obtient son Bachelor of Arts en 1904, à tout juste 16 ans - il est le plus jeune et le major de sa promotion. Impressionnés par ses facultés hors normes, ses professeurs, dont la plupart sont européens, le poussent à poursuivre ses études en Angleterre. Cependant la migration d'un indigène est soumise au blanc-seing du Civil Surgeon qui, dûment consulté, oppose un veto formel : la santé fragile de Râman est incompatible avec le climat anglais. II reste donc en Inde. II n'en sortira, pour la première fois, qu'à l'âge de 33 ans.

\section{Formation}

Pour ses études universitaires, il choisit naturellement les sciences physiques. Son professeur R.L. Jones prend vite la mesure du formidable potentiel de Râman et décide de lui laisser une grande liberté : il le dispense d'assister aux cours magistraux et l'encourage plutôt à se former directement dans les livres, à son rythme, en quasi autodidacte. Râman, quoique très jeune, a assez de maturité pour saisir cette chance. II s'installe à la bibliothèque et dévore toutes sortes d'ouvrages, en ogre éclectique, méthodique et éclairé : il étudie quatre langues, il s'initie à l'histoire grecque et romaine ainsi qu'aux principes de la finance, et bien sûr il perfectionne sa maîtrise des mathématiques et de la physique théorique et expérimentale... Trois livres ne quittent plus son chevet et charpentent sa vision du monde: The Light of Asia, qui est une adaptation du sûtra décrivant la vie du Bouddha Shākyamuni selon la tradition Mahāyāna ; les Eléments d'Euclide, qu'il prend comme une initiation au grand mystère de la Nature; et surtout Die Lehre von den Tonempfindungen, un traité sur le son, de Hermann von Helmholtz. Ce dernier ouvrage est une révélation : Râman y découvre une vision de ce qu'est la recherche scientifique, et comment elle peut être menée. Son esprit, alors en pleine éclosion, assimile et adopte la pensée du grand maître allemand. Sous cette férule désincarnée, Râman met en œuvre, en totale autonomie, une véritable démarche scientifique : identification d'une problématique, recherche des réponses dans la littérature, choix d'un protocole expérimental, analyse des résultats 
- et même publication ! II applique cette stratégie pour ses premiers travaux, qu'il mène sur l'étude de la diffraction de la lumière par une ouverture asymétrique. Le professeur Jones lui ayant ouvert l'accès aux moyens (chiches) du laboratoire de physique, il met à profit son temps libre pour mener quelques expériences d'optique élémentaire. Avec une audace et une confiance remarquables, il soumet ses résultats au fameux et très respecté Philosophical Magazine de Londres, qui les accepte et les publie en 1906. Râman, alors âgé d'à peine 18 ans et pas encore diplômé, en est le seul auteur et il n'a aucun tuteur. C'est un tour de force - et à double titre, puisque son article est aussi la première publication au nom du Presidency College.

En janvier 1907, Râman décroche son Master of Arts avec les honneurs. Mais à cette époque, les débouchés en Inde sont rares, surtout en science. Sa santé fragile lui interdit toute migration pour l'Angleterre, et lui ferme les portes du très sélectif Indian Civil Service (ICS, I'organe le plus prestigieux du gouvernement). II se rabat, comme son frère Chandrashekhara Subrahmanya lyer avant lui, sur le Financial Civil Service (FCS), dont il est reçu premier au concours d'entrée de 1907. C'est aussi en 1907 qu'il rencontre celle qui deviendra Lady Râman. Lors d'une visite chez son ami Ramaswamy Sivan, qu'il connaît depuis la fac, Râman s'éprend de sa belle-sœur Lokasundari. Mais, bien qu'ils soient de la même caste Brahmane, ils appartiennent à des sous-castes différentes, ce qui en principe interdit toute union. Ce n'est que grâce à l'appui de son père, esprit ouvert et fervent progressiste, que Râman et Lokasundari parviennent à célébrer leur union. Le couple aura deux garçons, Chandrasekhar et Radhakrishnan. Lokasundari se révèle une compagne dévovée, consciente du génie de Râman et attentive à lui sanctuariser un espace d'intimité et de création. Dès son entrée au FCS, Ramân est nommé Assistant Comptable-Général de Calcutta, et le couple s'installe dans ce qui est alors la capitale de l'Inde britannique et la plus grande ville scientifique d'Asie.

\section{Prix Nobel}

Tandis qu'il est en poste à Calcutta, Râman pousse un soir la porte de I'Indian Association for the Cultivation of Science, qui a été fondée en 1876 par Mahendralal Sarkar [1833-1904] sur le modèle de la Royal Institution of Great Britain. II en devient un des membres les plus assidus, et s'y épanouit littéralement en donnant libre cours à sa créativité scientifique. II étudie l'acoustique des instruments de musique, notamment ceux à cordes frottées ainsi que les tablas et mridangams indiens. En dix ans, il se bâtit ainsi une solide réputation de physicien aussi bien en Inde qu'en Europe. Plusieurs étudiants se placent spontanément sous sa férule. Ses travaux finissent par attirer l'attention du chancelier de I'University of Calcutta, qui lui propose le poste de professeur de physique. Immédiatement séduit, Râman décide de renoncer à sa position au FCS, pourtant prestigieuse et pleine de promesses, et entame à 30 ans une carrière académique. Son élection rapide au rang de Fellow de la Royal Society de Londres, en 1924, le confortera dans son choix.

En 1921, Râman est invité à une conférence à Oxford, où il doit représenter l'University of Calcutta. Cette fois, pas de veto qui tienne : il se rend en Angleterre! Durant son voyage, faisant preuve d'une énergie inépuisable et d'une curiosité tous azimuts, il imagine, et mène à terme, quelques expériences simples sur la couleur de l'océan. Il publie ainsi une brève dans la revue Nature où il attribue le bleu de la mer à un processus de diffusion optique et non, selon la croyance populaire, à la réflexion du ciel. Cette première étude est suivie, dès son retour en Inde, d'une série de travaux sur la diffusion moléculaire optique, dont la plupart avortent ou se heurtent à des phénomènes inexpliqués. Ce n'est qu'en 1927, lorsqu'Arthur Holly Compton obtient le prix Nobel pour sa découverte de la diffusion inélastique des photons $X$ par la matière, que Râman fait enfin le lien : la clef de ses propres expériences ne serait-elle pas l'analogue optique de l'effet Compton? Avec son collègue K.S. Krishnan, il reprend l'intégralité de ses

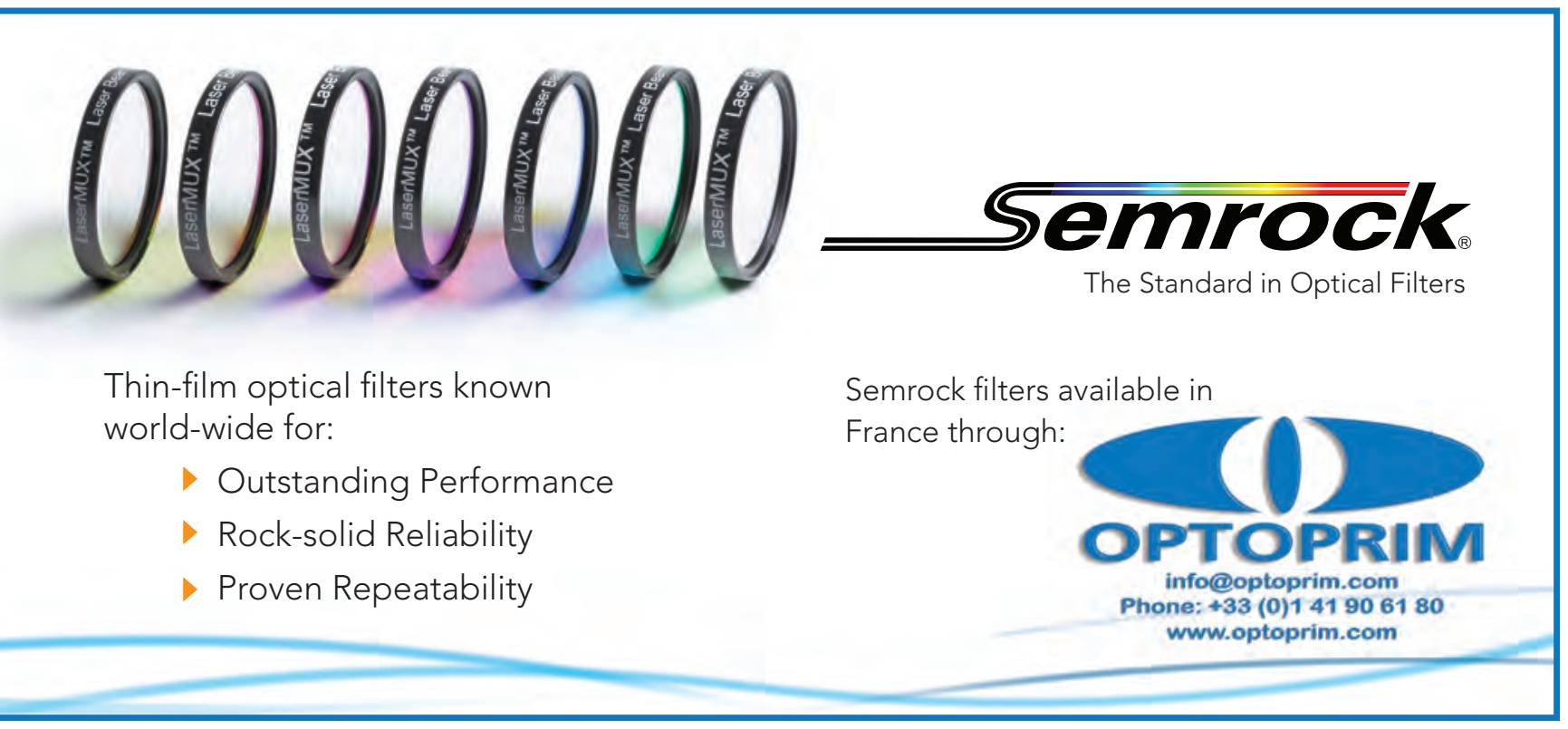


expériences avec cette nouvelle grille de lecture. Leurs travaux aboutissent le 28 février 1928 à la découverte de l'effet Râman. En mémoire de cette découverte, cette date est désormais célébrée chaque année comme l'Indian National Science Day. Notons toutefois que, bien que l'histoire ait attaché le nom de Râman à la diffusion inélastique de photons, cet effet physique est découvert indépendamment, et quasi-simultanément, par deux chercheurs soviétiques, Grigory Landsberg et Leonid Mandelstam.

Râman est fait chevalier de l'empire britannique en 1929, et en 1930 il devient le premier Asiatique et le premier Indien à recevoir le prix Nobel de physique. En 1933, il est nommé directeur de I'Indian Institute of Science, qui a été fondé en 1909 à Bangalore pour mener des recherches originales et promouvoir la formation en science et en ingénierie. Il est le premier non-Britannique à occuper ce poste. En 1947, il devient le premier National Professor d'une Inde qui vient d'accéder à l'indépendance. II démissionne de l'Indian Institute of Science en 1948 pour fonder, dès l'année suivante, le Râman Research Institute à Bangalore dans l'état du Karnataka - qu'il dirige jusqu'à sa mort.

\section{Ultima verbae}

Il reçoit de nombreux honneurs, dont le Bharat Ratna lla décoration civile la plus prestigieuse en Inde) en 1954 et le Prix Lénine pour la paix en 1957. Farouchement attaché au prestige de l'Inde, il décline une proposition de poste à l'université de Cambridge. Pourtant, dans les années 60 , Râman éprouve une profonde amertume devant ce qui ressemble à un complexe $\mathrm{d}$ 'infériorité de ses confrères indiens, qui recherchent plus volontiers l'inspiration en Occident qu'à l'intérieur des frontières du pays. $\mathrm{Ce}$ sentiment se confirme lorsque les autorités, visiblement convaincues que le progrès ne peut venir que d'ailleurs, décident $d$ 'investir lourdement dans de grosses installations techniques conçues par l'Europe ou les États-Unis. Outre la blessure d'amour-propre, cette stratégie s'oppose à la conviction profonde qu'a Râman que toutes les expériences, même les plus simples, peuvent mener à de grandes théories scientifiques. Malgré (et peut-être en réaction à) ce pessimisme ambiant, il reste formidablement actif, véritable locomotive à la tête de l'Académie des Sciences Indiennes et du Râman Research Institute. Toujours plein d'humour, merveilleux pédagogue, il sait susciter l'engouement de ses troupes.

Râman donne son dernier Gandhi Memorial Lecture le 2 octobre 1970. À la fin de l'exposé, au moment des questions, épuisé, il demande à poursuivre la séance en étant assis. II va bientôt célébrer ses 82 ans. Quelques jours plus tard, les valves de son cœur cèdent et il s'écroule dans son laboratoire. II survit encore quelques jours, le temps de mettre en ordre le Râman Research Institute et de laisser ses dernières consignes. II décède au petit matin du 21 novembre 1970.

\section{Références}

[1] C.V. Râman, A Pictorial Biography, compiled by S. Ramaseshan and C. Ramachandra Rao, Indian Academy of Sciences (1988).

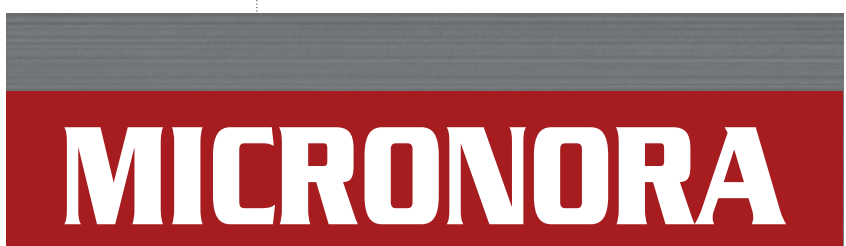

SALON INTERNATIONAL DES MICROTECHNIQUES

Multi-technologies et haute précision

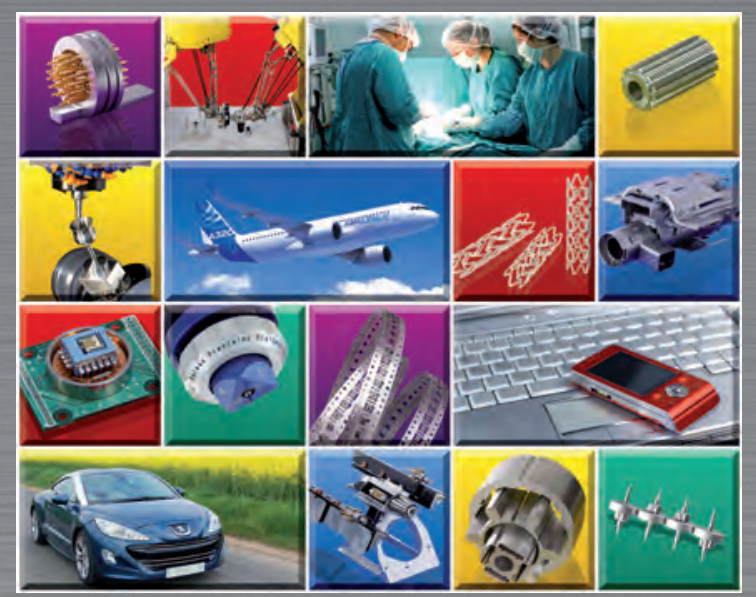

Plus petit, Plus précis, Plus intelligent

Découpage, Découpage fin

Usinage, Micro-usinage, Outillage

Décolletage de précision

Assemblage, Micro-assemblage

Automatisation, Robotique

Injection, Surmoulage

Métrologie, Mesure, Contrôle

Microfabrication, Nanotechnologie

Interconnexion, Packaging microélectronique

Traitement de surface, Ingénierie

Technologies de production ...

\section{3 - 26 septembre 2014}

\section{Besançon - France}

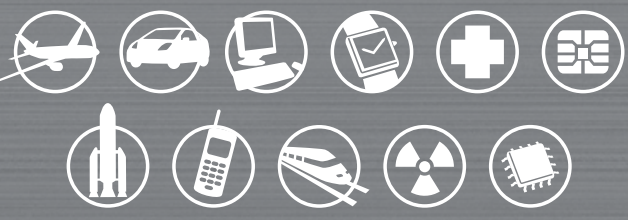

www.micronora.com

CS 62125 - 25052 BESANÇON Cedex - Tél. +33 (0)381 521735 